\title{
Overgranulation Masquerading as Recurrence of Malignant Melanoma in an Inguinal Lymphadenectomy Wound
}

\author{
Anais Rosich-Medina, Pratap Dutta, Damir Kosutic, Kantappa Gajanan* \\ Department of Plastic and Reconstructive Surgery, Christie Hospital NHS Foundation Trust, Manchester, UK \\ Email: ^Kantappa.Gajanan@chrisite.nhs.uk
}

How to cite this paper: Rosich-Medina, A., Dutta, P., Kosutic, D. and Gajanan, K. (2018) Overgranulation Masquerading as Recurrence of Malignant Melanoma in an Inguinal Lymphadenectomy Wound. Modern Plastic Surgery, 8, 45-49.

https://doi.org/10.4236/mps.2018.83006

Received: January 29, 2017

Accepted: July 2, 2018

Published: July 5, 2018

Copyright () 2018 by authors and Scientific Research Publishing Inc. This work is licensed under the Creative Commons Attribution International License (CC BY 4.0).

http://creativecommons.org/licenses/by/4.0/

\begin{abstract}
This case report discusses a patient with stage $3 \mathrm{~A}$ malignant melanoma who developed a large, friable mass at the site of a completion inguinal lymphadenectomy following a positive sentinel lymph node biopsy. We highlight the importance of considering both local disease recurrence and overgranulation in the differential diagnosis in order to guide appropriate management.
\end{abstract}

\section{Keywords}

Malignant Melanoma, Overgranulation

\section{Introduction}

The mechanisms involved in each of the three phases of wound healing rely on the complex interplay between a wide range of cells, inflammatory mediators and chemicals that eventually lead to epithelialisation of a wound. Although the three phases 1) haemostasis and acute inflammation, 2) proliferation and 3) remodelling and maturation overlap significantly, they should progress in an orderly manner [1]. Infrequently however, the interplay between the crucial factors of wound healing may become unbalanced and lead to an overgranulating wound.

Multiple factors lead to the formation of overgranulation and these can be subdivided based on the causative agents. Vuolo classified overgranulation into three types; Type I: inflammatory with excessive exudate due to continued minor trauma, friction or foreign body irritant such as dressings and drains; Type II: occluded wound environment (for example a hydrocolloid dressing) possibly due to infection or chronic colonisation; and Type III: cellular imbalance related 
to metalloproteinase enzymes that play an important role in the proliferative phase of wound healing [2]. Recognising the formation of overgranulation, its type and contributory factors are crucial in the management of surgical wounds particularly in those patients undergoing surgery for aggressive skin malignancies such as malignant melanoma where overgranulation could be mismanaged as a recurrence.

\section{Case Report}

A 65-year-old man with no significant past medical history was diagnosed with malignant melanoma (MM) on the left lower back. Histologically the MM had a Breslow thickness of $6 \mathrm{~mm}$, no ulceration, a mitotic rate of $4 / \mathrm{mm}^{2}$, lymphovascular invasion and was staged as pT4a. He underwent a wide local excision of the melanoma scar and sentinel lymph node biopsy (SLNB) of three sentinel nodes in the left axilla and one in the left groin. The left groin lymph node was the only positive node for metastatic melanoma. A staging computer tomography scan showed no evidence of distant metastasis. The patient then underwent a completion lymphadenectomy of the left groin and nine lymph nodes demonstrated no residual malignancy.

Post-operatively he developed lymphoedema of the limb and a wound infection of the left groin requiring frequent dressing changes. A stoma bag was placed to collect the serous fluid discharging from the wound. Approximately 2 weeks post-operatively an exuberant mass of spongy, friable tissue was noted around the edge of the wound where the stoma bag had been placed (Figure 1). The unhealthy looking friable mass of tissue, which did not bleed readily, raised the possibility of MM recurrence. The friable mass was excised in the outpatient department and sent for histopathology, which reported it as granulation tissue with bacterial overgrowth and suppurative exudate. There was no evidence of malignancy.

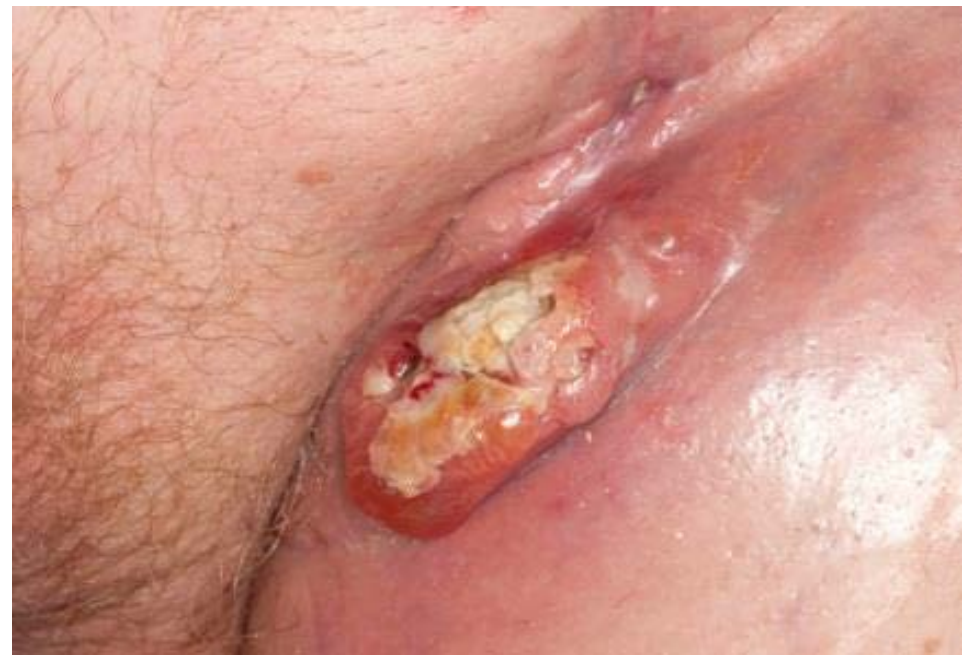

Figure 1. Large, friable mass at the site of a left inguinal completion lymphadenectomy wound 2 weeks post-operatively. 
The wound was managed with silver based dressings, Aquacel Ag (Figure 2(a) and Figure 2(b)), for several weeks and completely healed by 6 weeks (Figure 3). The patient was referred to the lymphoedema specialist nurses and his symptoms have improved. He is currently undergoing three monthly follow up appointments as per the United Kingdom guidelines for malignant melanoma.

Full informed signed consent was obtained from the patient for the use of his clinical information and medical photographs in this case report.

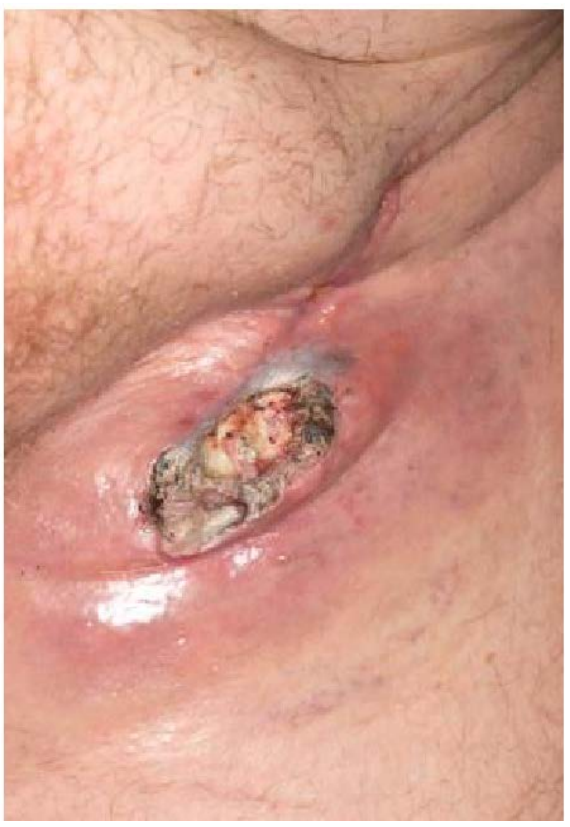

(a)

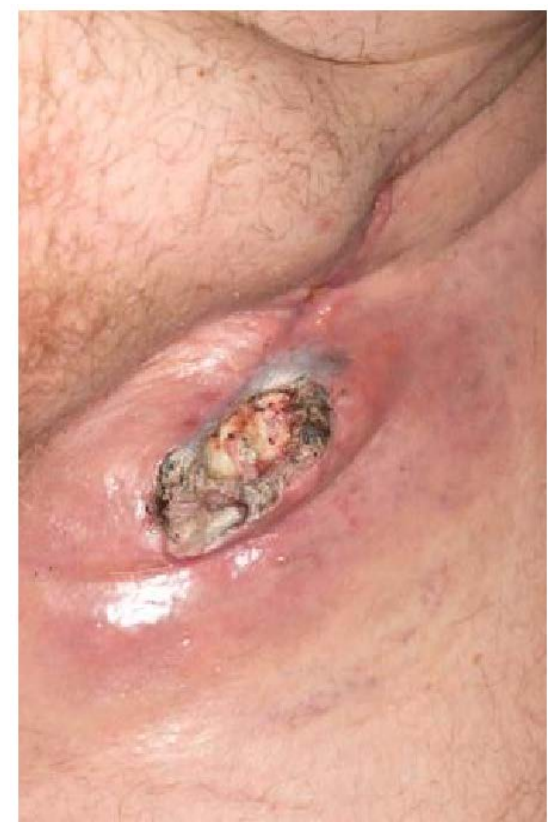

(b)

Figure 2. (a) and (b) 1 week post-excision of large friable mass and treatment with silver nitrate and Aquacel Ag dressings.

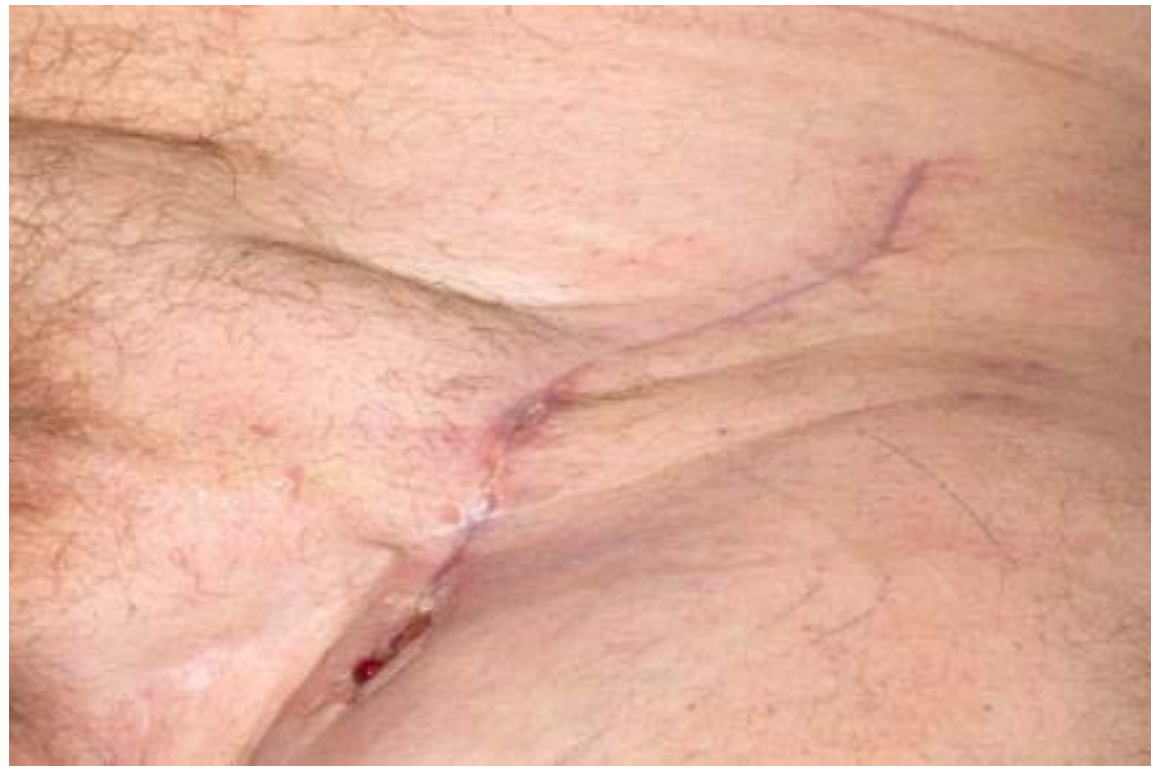

Figure 3. 6 weeks post treatment for overgranulation. 


\section{Discussion}

It is vital that a malignant recurrence is considered and excluded in the setting of post surgical wound healing complications in patients treated for aggressive skin malignancies and not merely treated as overgranulation without histological confirmation [3]. A thorough history, comprehensive wound assessment and general examination of the patient was crucial in this case to reach a diagnosis. Taking a biopsy of the suspicious lesion will help confirm or refute the clinical impression. The patient hereby presented had an aggressive malignant melanoma stage 3A, T4aN1M0. Although he underwent complete surgical clearance and regional disease control he had a high risk of disease recurrence with a 5 year survival rate of $63 \%$ [4]. The comparatively shorter time period between surgery and presentation of the friable growth at the wound site made the possibility of recurrence unlikely but not implausible. Several causative factors were elicited from the patients' history that directed the clinical suspicion towards overgranulation, namely, wound infection, oedema and the occlusive dressing (stoma bag) over the wound. Overgranulation associated with infection is best treated with antibacterial dressings such as silver, honey and iodine to reduce local bacterial colonization [5]. Cautious use of silver nitrate precipitates the inflammatory process of wound healing and can be useful in treating overgranulating wounds. The causative factors were removed and appropriate dressings used for the management of overgranulation in this patient and he responded well to treatment. Clinicians should be aware of the possibility of developing overgranulating surgical wounds and the need for obtaining histological confirmation to exclude malignancy, particularly in patients with aggressive malignancies.

\section{Conclusion}

Overgranulation is an important complication of wound healing and can be mistaken for recurrence in the background of skin cancer surgery. A thorough clinical history and examination will guide the clinician towards the correct diagnosis and histological confirmation will ensure that the appropriate management is instituted.

\section{Conflict of Interest}

The authors claim no conflict of interest for this work.

\section{References}

[1] Broughton, G., Janis, J. and Attinger, C. (2006) The Basic Science of Wound Healing. Plastic and Reconstructive Surgery, 117, 12S-34S. https://doi.org/10.1097/01.prs.0000225430.42531.c2

[2] Vuolo, J. (2010) Hypergranulation: Exploring Possible Management Options. British Journal of Nursing, 19, S6-S8

[3] Young, T. (1995) Common Problems in Wound Care: Overgranulation. British 
Journal of Nursing, 4, 169-170. https://doi.org/10.12968/bjon.1995.4.3.169

[4] Romano, E., Scordo, M., Dusza, S.W. and Coit, D.G. (2010) Site and Timing of First Relapse in Stage III Melanoma Patients: Implications for Follow-Up Guidelines. Journal of Clinical Oncology, 28, 3042-3047. https://doi.org/10.1200/JCO.2009.26.2063

[5] Hampton, S. (2007) Understanding Overgranulation in Tissue Viability Practice. British Journal of Community Nursing, 12, S24-S30.

https://doi.org/10.12968/bjen.2007.12.Sup4.43000 